\section{Optimisation of Fuel Usage and Steam Availability in the Power and Steam Plant of a Paper Mill.}

\author{
PAUL AUSTIN ${ }^{1}$, MATTHEW McEWAN ${ }^{2}$, JOHN \\ MACK $^{2}$, JOHN GODSAL ${ }^{3}$, JOHN TYLER $^{3}$ \& JAN \\ MACIEJOWSKI ${ }^{1}$ \\ ${ }^{1}$ Cambridge University Engineering Dept, Trumpington St., \\ Cambridge $\quad$ CB2 $\quad 1 T L, \quad$ ENGLAND; \\ $\{$ pca23,jmm1\}@cam.ac.uk
}

${ }^{2}$ Perceptive Engineering Ltd, Daresbury Innovation Centre, Keckwick Lane, Darebury, Runcorn WA4 4FS, ENGLAND http://www.perceptiveapc.com;

${ }^{3}$ Aylesford Newsprint Ltd, Newsprint House, Bellingham Way, Aylesford, Kent ME20 7DL, ENGLAND

KEYWORDS: Model Predictive Control, Improved Efficiency, Optimisation, Power and Steam Supply System

\section{ABSTRACT}

This paper presents the results of a project aimed at minimising fuel usage while maximising steam availability in the power and steam plant of a large newsprint mill. The approach taken was to utilise the better regulation and plant wide optimisation capabilities of Advanced Process Control especially Model Predictive Control (MPC) techniques. These have recently made their appearance in the pulp and paper industry but are better known in the oil and petrochemical industry where they have been used for nearly 30 years.

The issue in the power and steam plant is to ensure that sufficient steam is available when the paper machines require it and yet not to have to waste too much steam when one or more of the machines suffers an outage. This is a problem for which MPC is well suited. It allows variables to be kept within declared constraint ranges, a feature which has been used, effectively, to increase the steam storage capacity of the existing plant. This has resulted in less steam being condensed when it is not required and in significant reductions in the need for supplementary firing.

The incidence of steam being dump-condensed while also supplementary firing the CHP plant has been reduced by $95 \%$ and the overall use of supplementary firing is less than $30 \%$ of what it was. In addition the plant runs more smoothly and requires less operator time. The yearly benefit provided by the control system is greater than $£ 200,000$, measured in terms of 2005 gas prices.

\section{INTRODUCTION}

Increases in the cost of oil and gas over the last few years have renewed the focus in most European paper mills on ways to save energy. Attention at Aylesford Newsprint Ltd (ANL) turned to the power and steam plant, represented in Figure 1, which supplies steam to three paper machines.

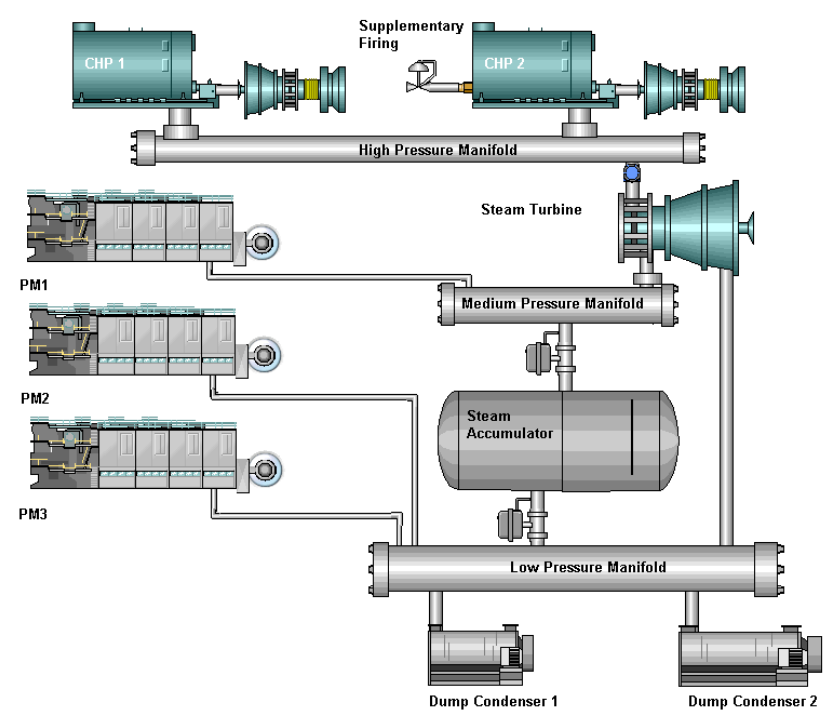

Figure 1: The Aylesford Newsprint Power and Steam Plant

Steam is supplied to the high pressure manifold via two CHP gas turbines with heat recovery; supplementary firing on CHP 2 provides additional steam above base load demand. The High Pressure manifold, nominally kept previously at 60 bar, supplies the medium pressure manifold (nominally operated at 14 bar), through a steam turbine that can be used to raise more power, and whose exhaust feeds steam to the low pressure manifold. Steam can also be passed from the HP manifold to the MP manifold through a sequence of Pressure Reducing Desuperheating Stations (PRDSs). The medium pressure manifold supplies steam to one of the three paper machines; the low pressure manifold, which operates nominally at 4 bar, supplies the other two paper machines. A steam accumulator supplied from the medium pressure manifold provides steam capacity to the low pressure manifold. Excess steam can be bled from the system through one or both of two dump condensers, which are attached to the LP manifold.

An internal study revealed several possibilities for energy saving:

- There were known to be occasions when supplementary firing was being used while steam was being dumped through the condensers

- The capacity of the steam accumulator and indeed of the pressure manifolds could be better utilized for storing steam if the manifolds could be run between upper and lower limits rather than just to a fixed setpoint.

- There was seen to be an opportunity to optimize in real time the ways in which steam is passed from the HP to the LP manifold.

ANL decided to investigate the potential for Advanced Process Control (APC) to provide better real time control and optimization of the power and steam system. Mill staff were very familiar with APC technology, having already employed it on their de-inking plant, on their newsprint machines and on their sludge combustor.

Using Model Predictive Control (MPC), the core 
technology of APC, the multivariable character of a process is quantified within a model which is then used to calculate optimal controller moves. The multivariable model makes it possible to predict the effects of all inputs, control inputs and disturbances alike, on the output variables. This allows the controller to make coherent use of all the control variables to achieve better regulation and optimising control, while maintaining the variables within their specified constraint ranges.

This paper outlines APC work with the ANL power and steam plant. APC implementations on ANL's paper machines [1, $2 \&$ 3] and de-inking plants [4] is reported elsewhere. Within the pulp and paper industry, Perceptive Engineering Ltd (PEL) is also working in pulp mills (kraft and mechanical pulping) and sludge combustion.

\section{ADVANCED PROCESS CONTROL CONCEPTS}

A robust Advanced Process Control (APC) application requires a toolset that offers a wide range of $\mathrm{APC}$ technologies. PEL's APC software toolset includes MPC, modelling and control using Artificial Neural Networks, Adaptive Control, Inferential Estimation and Fuzzy Logic. All of these tools are available in the ControlMV package, developed and used in industry applications by PEL.

MPC, the core technology of most APC toolsets, is now overviewed. Much more complete descriptions of APC techniques can be found for example in [5] and [6]. The objective of the MPC controller is to determine a sequence of control moves, starting with the current control move and continuing with control moves that would be applied at some time in the future. This series of 'ideal' control moves is calculated in such a way as to ensure that one or more controlled (output) variables move to their setpoints in an optimal manner.

The control calculation at each point in time is based on the current value of each controlled variable, as well as a prediction of the future values of each controlled variable. This approach differs from the classical PID approach to control, in which only the current control move is calculated based on the current setpoint error. The prediction of the future values of each controlled variable is based on an explicit model of the process. This model encapsulates all of the multivariable 'cause and effect' dynamic relationships between controlled variables, manipulated variables (control inputs) and feedforward variables (measured disturbance variables arising in upstream process elements).

The control algorithm typically uses a Receding Horizon approach. In this approach, the future variation of the controlled variables is predicted up until a finite time in the future (called the 'control horizon'). A sequence of control moves is calculated for each manipulated variable, at each execution interval from the current time up to the control horizon. Only the first move in this sequence is actually implemented, and at the next execution interval, the entire control calculation is repeated. The repetition of the control algorithm at each execution interval provides a 'negative feedback' mechanism which minimises the effect of unmeasured disturbances and inaccuracies in the model.
Generally MPC is applied to multivariable systems having a number of manipulated variables each affecting more than one of the controlled variables. However, for a system involving a single manipulated (control) variable and a single controlled (output) variable, the following diagram illustrates the methodology behind most MPC-based controllers.

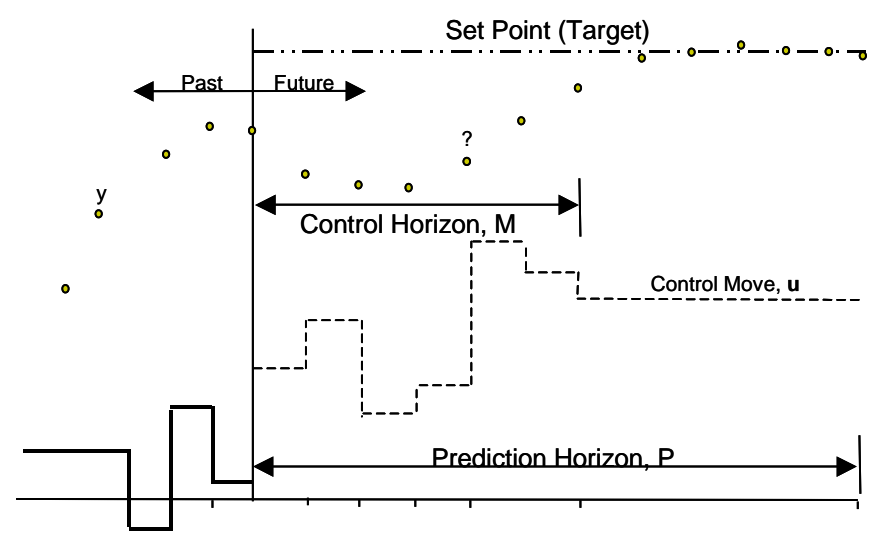

Figure 2. Control Strategy of Most MPC Controllers

MPC is a particularly advantageous control strategy for processes having any of the following characteristics:

- Processes whose system dynamics are highly coupled and multivariable; that is, processes in which moving a particular input variable affects more than one controlled variable. Because of the multivariable process model at its centre, MPC is ideally suited to multivariable problems and is able to provide optimal control for almost all situations.

- Processes whose system dynamics are long or complex and in particular, when they have long dead times, and/or inverse (that is, non-minimum phase) responses. Plants with characteristics like these are hard to control using PID techniques PID controls usually need to be detuned. However using MPC, the explicit process model can predict the effect of complex process dynamics and calculate control moves accordingly.

- Processes where for optimum operation, the controlled variables need to be kept close to operating limits. With MPC, controlled variables can be maintained either at a setpoint (in the same way as with traditional PID control), or they can be kept within defined upper and lower constraint limits. Constraint control allows a controlled variable to move freely unless the variable is predicted to move outside of its constraint limits within the control horizon. If a constraint violation occurs, then the MPC will re-calculate control moves to ensure the controlled variable is maintained within limits in an optimal manner.

- Processes having feedforward disturbances that can be accurately measured. The use of an explicit process model means that the effect of measured disturbances from upstream processes can be used to predict the subsequent future variation of one or more controlled variables. Given this prediction, 
the MPC controller will calculate control moves in order to minimise the effect of this disturbance.

- Processes in which it is desirable to control to a variable that is measured intermittently, often by lab measurements. Often variables that are critical to quality can only be measured in this offline way. By using model-based inferential sensors, it is possible to control to the inferred quality value in between lab measurement updates. This can considerably reduce the standard deviation of critical-to-quality variables.

In general, if any one of the above process conditions exists, then there are good grounds for considering MPC as a process improvement tool.

The controller design process is typically based around an investigation of the process, taking into account a number of factors including:

- The economic benefit associated with the tighter control of key variables.

- Bottlenecks and limitations in the process equipment, including safety requirements.

- The state of current process instrumentation.

- The performance of the existing regulatory control systems.

- The knowledge and problems experienced by operators and process staff.

The investigation of the process will typically also involve process tests in order to determine the dynamics of the process. The final design of the advanced control system should encapsulate all of the factors listed above.

\section{PLANT OPERATING OBJECTIVES}

The main purpose of the power and steam plant (see Fig 1) is to provide steam to the three paper machines. A secondary purpose is to generate power from the steam that has to be raised to meet the steam demand. The main cause of disturbance to the system arises from mill outages and sheet breaks, which typically happen with very little or no prior warning, and which cause sudden and often very large instantaneous changes in steam demand.

When all the paper machines are running and using steam:

- Base load running of the CHP units generates insufficient steam so some supplementary firing of CHP 2 is required.

- There should be no flow through the steam condensers. However, the operators typically used supplementary firing to ensure sufficient steam was available, even while steam was being dumped.

When paper machine outages or sheet breaks occur:

- The CHP base load supplies excess steam.

- Supplementary firing is not required

- The steam condensers need to be used to remove excess steam from the system.

The typical effect of a sheet break on the steam supply system is shown in Figure 3.

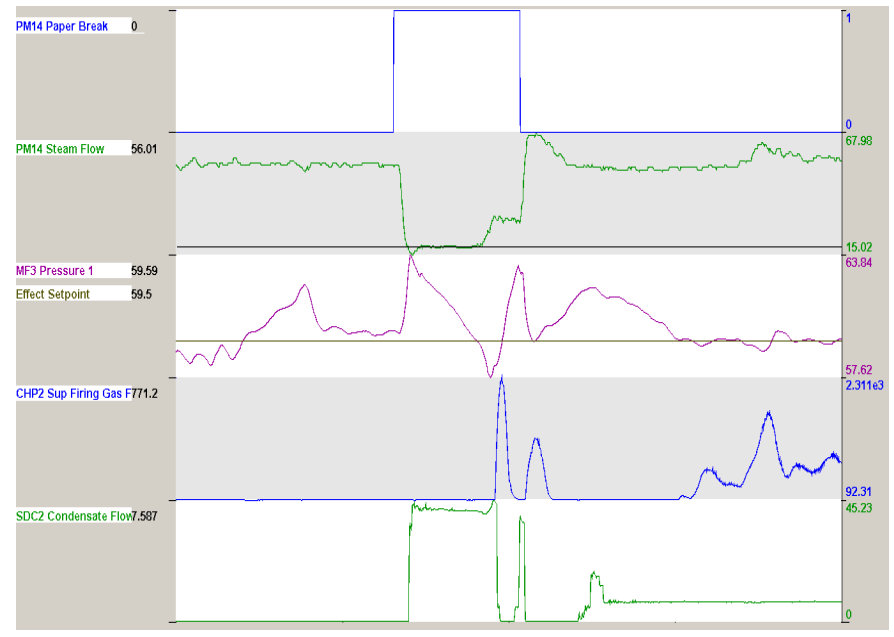

Figure 3: Effect of a Paper Break on Steam Supply System

When the paper break occurs, the steam flow to the mill starts to drop off steeply. The third trend of Fig 3 shows the effect on the HP steam header pressure: it rises initially but in response to the operators' having opened the steam condenser valves, the HP pressure begins to return towards target. However, too much steam was dumped (fifth trend) and the HP manifold pressure drops below target, requiring supplementary firing to be used (fourth trend).

Considering this pattern of operation, ANL sought an optimizing power and steam control system that could:

- Better manage the use of steam condensation and supplementary firing during load changes, so as to minimise energy wastage

- Utilise the steam storage capacity in the network, so that excess steam could be stored when the steam load is less than maximum and discharged during peak periods when insufficient steam is being produced. This calls for the ability to operate the steam accumulator and steam header pressures in a constrained range rather than just to a setpoint.

\section{APC SYSTEM DESIGN}

A supervisory, optimizing Advanced Process Control (APC) system was designed for the ANL power and steam plant. Design considerations included the following:

- Safe operation was a high priority for the APC system: expensive equipment in the steam network needed to be protected, especially including the steam turbine.

- Pressure limits should not be exceeded since safety systems would then automatically vent excess steam, thus wasting energy

- It is crucial to ensure steam is available to meet paper machine demand: lost production is more expensive than energy.

- The steam network is a highly multivariable system: the state of each header is affected by all the elements feeding it and drawing from it and therefore control changes at the low pressure end will quickly affect the high pressure end and vice versa.

It is clear therefore that the problem of controlling steam 
header pressures needs to be seen as a problem involving the whole system rather than merely a problem of designing individual steam pressure control loops.

A multivariable input output representation of the power and steam supply system as modeled by the APC system is shown in Figure 4.

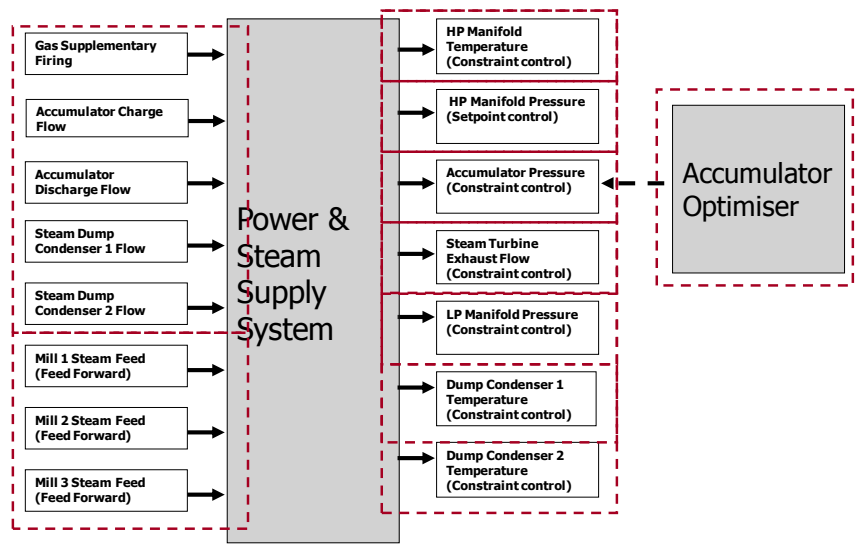

Figure 4: Variables Within the Scope of the APC System

\section{Features of the APC Solution}

The new APC control system has been designed to maximize steam availability while minimizing gas usage. The new system incorporates as much as possible of the previous existing regulatory control network: the existing turbine controllers, PRDS controllers, safety valves, steam condenser temperature and bank controllers were all left in place and the system was modeled and characterized with these loops operative. The APC system has been set up so as to minimize supplementary firing and steam dump condensing at all times, and in particular, to ensure that steam is never condensed at the same time as supplementary firing is being used.

Fig 4 shows that the new APC system is responsible for operating:

- Supplementary firing

- The steam accumulator

- The steam dump condenser - control of this was previously in the hands of the operators

Of the input variables shown in Figure 4:

- all five Manipulated Variables (MVs) represent supervisory setpoints determined by the APC system and written to local flow controllers to implement. The APC system is tuned to keep these flows as low as possible.

- the other three input variables, the Feedforward Variables (FFs), represent the disturbance imposed on the steam supply system by variable rates of steam use at the three paper machines.

Of the Controlled Variables (CVs):

- the HP manifold pressure is the most important; it is affected by all the MVs. It is controlled to setpoint, which is allowed to vary between upper and lower limits specified by operations personnel.

- Steam condenser temperatures are controlled within constraints to prevent vapour locking.
- The steam turbine exhaust flow is maintained with constraints to prevent turbine damage.

The steam accumulator optimizer manages the accumulator pressure (analogous to steam 'level'), allowing it to act as though it were an additional low pressure boiler. The accumulator optimizer:

- charges the accumulator when excess steam is being produced, thus reducing the amount of steam that has to be dumped

- discharges the accumulator when supplementary firing would otherwise be above the minimum level.

The controller has been implemented using PEL's ControlMV toolset, in which MPC is implemented using a quadratic cost function. Other features of the design are as follows:

- $\quad$ The specified constraints have been enforced using QP with LR simulation. That is, for a soft constraint variable, the model is used to project ahead to find whether a constraint is violated. If it is, then the CV variable is converted to a setpoint type whose target value is adjusted to prevent the variable violating the declared constraint.

- The system is modeled using linear ARX models of first and second order. As indicated in Fig 4 there are $5 \mathrm{MVs}, 3 \mathrm{FFs}$ and $7 \mathrm{CVs}$. To meet the process requirements described above, the steady state target weights of all $5 \mathrm{MVs}$ are set towards zero. Of the CVs, all but the HP manifold pressure and the accumulator pressure are managed as soft constraints. The HP manifold pressure is a hard constraint and the accumulator pressure is determined by the optimiser and managed as a setpoint.

- Only the accumulator pressure is explicitly 'optimised'. The optimal point of the other variables is found as a result of the controller tuning in which, by virtue of the fact that all the MVs have zero steady state target weight, the MVs are gently 'pulled' to the closed position. This means that when supplementary firing is in use, the tuning tries slowly to remove supplementary firing with the effect of running with a slightly lower HP pressure. Also, when steam is being dumped, the tuning attempts to reduce the amount of dumping, which has a tendency to raise the HP manifold pressure slightly. This enforces an important known feature of the cheapest solution.

- The controller runs at a 20 second time interval with a 20 step horizon. Solution time is negligibly small.

\section{Behaviour of Steam Supply System Under APC Control}

Figure 5 shows an example of the response of the system after a sheet break under APC control. The steam stored in the accumulator (third trend) is sufficient to enable the HP pressure (first trend) to recover without recourse to 
supplementary firing (second trend) after the sheet break (effect on steam demand shown in sixth trend) and the consequent dumping (fifth trend) of steam.

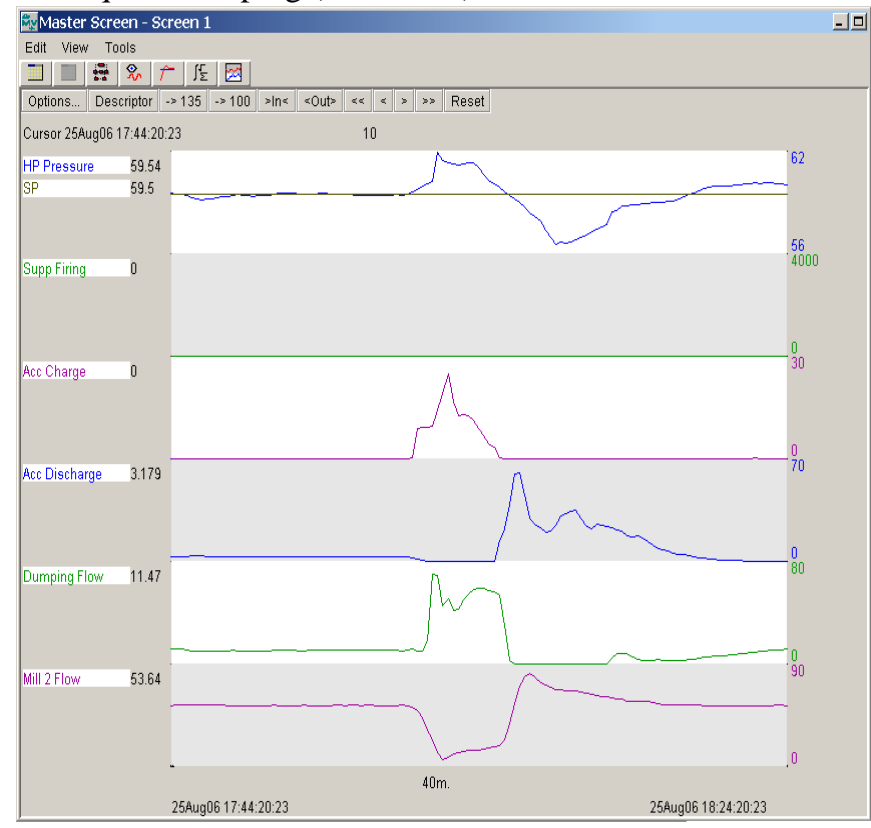

Fig 5: A Sheet Break Requiring no Supplementary Firing

Figure 6 shows another situation in which the APC system manages steam flows and pressures through a period including a machine outage. In the first half of the plot, during which all three machines are operating and there is insufficient steam from the base load CHP supply to meet demand, the optimizer discharges the accumulator, avoiding the need for supplementary firing. When a machine outage occurs, the optimizer manages the discharge of steam while re-charging the accumulator. Once all three machines are running again, steam demand is able to be met without supplementary firing. In the last quarter of the plot, the accumulator discharge ceases when the pressure reaches the low constraint.

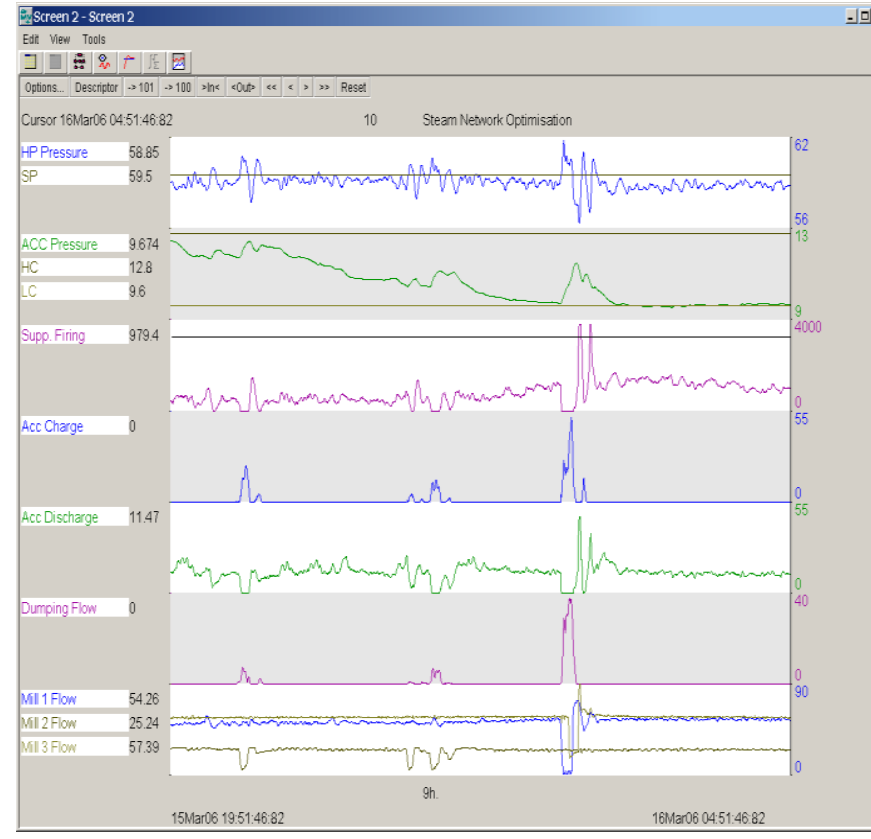

Fig 6: Steam Optimisation During a Second Sheet Break

\section{APC PERFORMANCE BENEFITS}

The APC system has delivered some significant benefits for the power \& steam plant:

- Overall, there has been a reduction of $70 \%$ in the use of supplementary firing

- There has been a reduction of $95 \%$ in the incidence of dump-condensing steam whilst also burning gas for supplementary firing

- A big contributor to these benefits is the optimization of the accumulator, a new feature of operation of the system

- The APC system has resulted in smoother operation of the power \& steam plant and reduced operator workload.

Based on 2005 gas prices (before the onset of the more recent hike in gas prices), the total yearly benefit, projected from the first three months of operation was expected to be well in excess of $£ 200,000$, giving a project payback time measured in a small number of months.

\section{CONCLUSIONS}

A new multivariable optimizing supervisory APC system has been installed on the power and steam plant at a large newsprint mill. This system has reduced supplementary firing to $30 \%$ of what it was and has nearly eliminated steam venting as a means of operating the system. This has been done by making better use of the capacity of the steam accumulator and steam headers by operating them between pressure limits rather than to setpoint. The benefits provide a very fast return on the project investment.

A similar approach can in principle be taken to any other steam network, no matter what its size and complexity. Higher energy costs are making the optimization of steam networks increasingly worthwhile. By virtue of its inherent character, APC is able to coordinate both the short term supervisory regulation issues and the longer term optimization issues of these plants.

APC appears to have considerable potential for performance improvement and cost reduction in power and steam plants and its adoption can be expected to develop rapidly over the next few years.

\section{ACKNOWLEDGEMENT}

The authors would like to thank Aylesford Newsprint Ltd for permission to present suitable plant information and data in this paper.

\section{REFERENCES}

1. Austin P.C., Mack J., Lovett D., Wright M. \& Terry M, "Improved Wet End Stability of a Paper Machine Using MPC”, Control Systems 2002, SPCI, Stockholm (2002)

2. Austin P.C, Mack J., Bauer A. and Marotte F., "Improved Wet End Stability and Performance using Multivariable Model Predictive Control and Optimisation at Papeteries de Clairefontaine" Proceedings ATIP Conference, Bordeaux, France (2004) 
3. Austin P.C., Mack, J. and McEwan M, "Increased Production and Improved Quality on Paper Machines using Advanced Process Control", Proc $61^{\text {st }}$ APPITA Conference (2007)

4. Austin P.C., Mack J., Gordon A., Smith M. and Astrom A., "Online Optimisation and Control of a De-inking Plant using Advanced Process Control" Proceedings of the I Chem E APC 7 Conference (2004).

5. Maciejowski J.M., "Predictive Control with Constraints", Prentice-Hall (2002).

6. Camacho E.F. and Bordons C., "Model Predictive Control", $2^{\text {nd }}$ ed, Springer (2004). 